\title{
Vitreomacular traction syndrome
}

\author{
Síndrome de tração vítreomacular
}

Thiago Gonçalves dos Santos Martins ${ }^{1}$, Thomaz Gonçalves dos Santos Martins ${ }^{2}$, Ever Ernesto Caso Rodriguez ${ }^{3}$, Ana Luiza Fontes de Azevedo Costa ${ }^{4}$

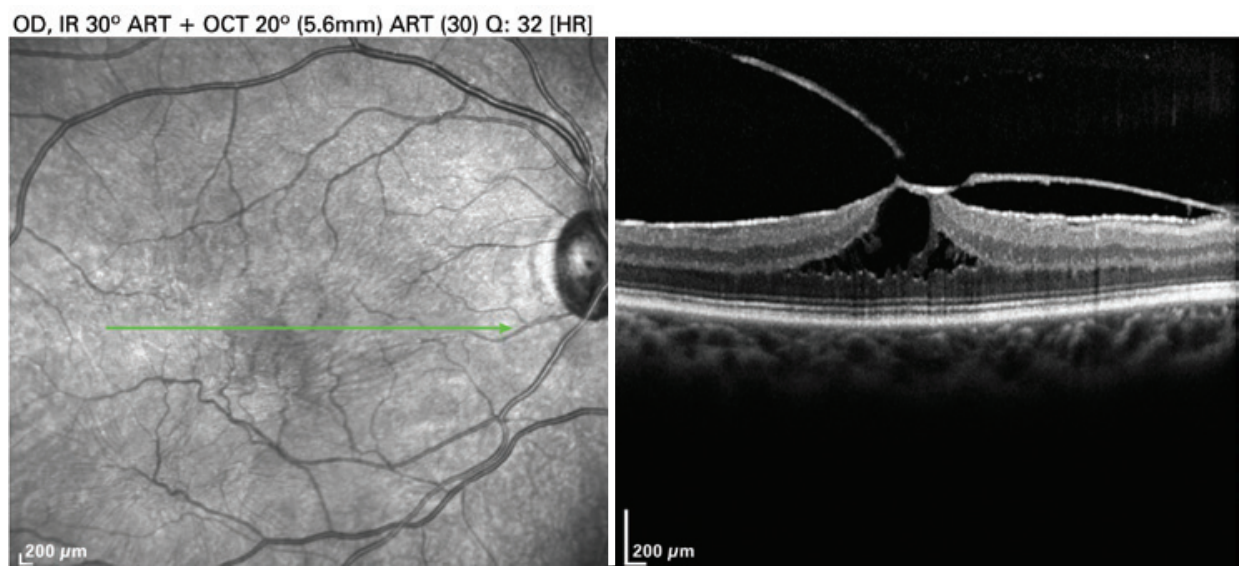

Figure 1. Camel-Hump Sign. Optical coherence tomography of the right eye showing centripetal traction area in macule region suggesting appearance of tractional retinal detachment

NRS, a woman, 71-year-old, who born in São Paulo, Brazil, sought our care service complaining of progressive low visual and metamorphopsia in the right eyes (RE) for 3 months. She had no-history of comorbidities, ophthalmologic surgeries or eye diseases.

Upon examination her best-corrected visual acuity was $20 / 80$ in the RE and 20/20 in the left eye. Funduscopy showed vitreous condensation in fovea region of the RE. Optical coherence tomography (OCT) showed vitreomacular traction due to partial posterior vitreous detachment that disorganized the fovea structure and formed an aspect of "camel-hump" associated with the presence of high-reflectivity membrane, generating moderate centripetal traction of the retina surface with preservation of external and internal lines of photoreceptors (Figure 1).

A vitrectomy was done with total vitreous detachment staining with triamcinolone for removal of posterior vitreous and macular epiretinal membrane with peeling of the limited internal membrane. An improvement in metamorphopsia was seen in the first week after surgery. Vitrectomy can present complications such as iatrogenic retinal rupture in $1.6 \%$ of cases and development of cataract in $69 \%$ of phakic patients within the 2 years after surgery. ${ }^{(1)}$ For this reason, some physicians have opted to intravitreal injection of ocriplasmin when

\footnotetext{
${ }^{1}$ Universidade Federal de São Paulo, São Paulo, SP, Brazil.

${ }^{2}$ Universidade Estácio de Sá, Rio de Janeiro, RJ, Brazil.

${ }^{3}$ Universidade de São Paulo, São Paulo, SP, Brazil.

${ }^{4}$ Hospital Federal dos Servidores do Estado, Rio de Janeiro, RJ, Brazil.

Corresponding author: Thiago Gonçalves dos Santos Martins - Universidade Federal de São Paulo, Rua Botucatu, 821, 2nd floor - Vila Clementino - Zip Code: 04023-062 - São Paulo, SP, Brazil Phone: (55 11) 5085-2010 - E-mail: thiagogsmartins@yahoo.com.br

Received on: Sep 16, 2013 - Accepted on: Jan 14, 2014

DOI: 10.1590/S1679-45082015AI2979
} 
vitreomacular traction is associated with macular hole of diameter less than or equal to 400 microns.

Vitreomacular traction syndrome is a vitreoretinal interface alteration where presence of myofibroblast associated or not to collagen layers prevents the total detachment between posterior vitreous and the retina. ${ }^{(2-4)}$

Ultrastructure of vitreoretinal interface present two basic standards: cellular layer adhered to limited internal membrane, and fibrocelular proliferation separated from the limited internal membrane by collagen layer, which is similar to idiopathic epiretinal membrane. In both groups, the predominant cellular type is miofibroblast that explain the high prevalence of cystoid macular edema and progressive vitreomacular traction. ${ }^{(3)}$

Structural changes in retina because of changes observed in OCT have complemented the diagnosis and support the management that is expected or not, to be adopted. Cases with good visual acuity and small distortion of retinal architecture present better prognosis. Posterior vitrectomy has been proposal in the first signals of prognosis worsening. Worsening metamorphopsia and visual acuity associated with funduscopy and tomographic images show distortions in retinal architecture resembling "mountain range" and "camel-hump", which suggest intervention. Anatomical improvement after surgery is not always followed by improves in visual acuity, so that reflecting probable functional changes and retinal microstructures that cannot be reestablished after surgery. However, after metamorphopsia an improvement is seen; which constitutes one of the main complaints of these patients.

The OCT constitutes an indispensable semiologic weapon in the diagnosis and treatment of vitreous traction syndrome. These images help in diagnosis and followup of patients.

\section{REFERENCES}

1. Jackson TL, Nicod E, Angelis A, Grimaccia F, Prevost AT, Simpson AR, et al. Pars plana vitrectomy for vitreomacular traction syndrome: a systematic review and metaanalysis of safety and efficacy. Retina. 2013;33(10):2012-7. Review.

2. Hikichi T, Yoshida A, Trempe CL. Course of vitreomacular traction syndrome. Am J Ophthalmol.1995;119(1):55-61.

3. Gandorfer A, Rohleder M, Kampik A. Epiretinal pathology of vitreomacular. traction syndrome. Br J Ophthalmol. 2002;86(8):902-9.

4. Doi N, Uemura A, Nakao K, Sakamoto T. Vitreomacular adhesion and the defect in posterior vitreous cortex visualized by triamcinolone-assisted vitrectomy. Retina. 2005;25(6):742-5. 